\title{
Cervical intraepithelial neoplasia in pregnancy: Interference of pregnancy status with p16 and Ki-67 protein expression
}

\author{
ANDREA CIAVATTINI ${ }^{1}$, FRANCESCO SOPRACORDEVOLE ${ }^{2}$, JACOPO DI GIUSEPPE ${ }^{1}$, LORENZO MORICONI ${ }^{1}$, \\ GUENDALINA LUCARINI ${ }^{3}$, FRANCESCA MANCIOLI ${ }^{1}$, ANTONIO ZIZZI $^{4}$ and GAIA GOTERI ${ }^{4}$ \\ ${ }^{1}$ Gynaecologic Section, Woman's Health Sciences Department, Marche Polytechnic University, I-60123 Ancona; \\ ${ }^{2}$ Gynecologic Oncology Unit, Department of Surgical Oncology, Oncologic Referral Centre, National Cancer Institute, Aviano, \\ I-33081 Pordenone; ${ }^{3}$ Histology Section, Department of Molecular Pathology and Innovative Therapies; \\ ${ }^{4}$ Pathological Anatomy Section, Department of Biomedical Sciences and Public Health, \\ Marche Polytechnic University, I-60121 Ancona, Italy
}

Received May 30, 2015; Accepted April 22, 2016

DOI: $10.3892 / \mathrm{ol} .2016 .5441$

\begin{abstract}
To date, there are evidence-based guidelines available for cervical dysplasia diagnosed in pregnancy. Certain functional biomarkers have proven useful in the prediction of regressing and non-regressing cervical intraepithelial neoplasia (CIN) lesions in non-pregnant women. In the present study, Ki-67 and p16 immunostaining were evaluated in different grades of CIN lesions diagnosed in pregnant or non-pregnant women with the aim to identify any differences in order to better understand the behavior of CIN in pregnancy. The current retrospective case-control study included 17 pregnant patients that conceived naturally with first-time onset of CIN occurring at no later than 16 gestational weeks. The control group included 17 non-pregnant patients matched for age, parity and number of previous sexual partners. Exclusion criteria included previous cervical treatment, immunocompromised status, chronic hepatitis B and/or C and cigarette smoking. p16 and Ki-67 protein expression were respectively detected using the CINtec Histology kit and monoclonal antibodies against Ki-67. p16 and Ki-67 staining were analyzed using a classification system based on the distribution of positivity on a semi-quantitative three point-scale. p16 and Ki-67 immune reactivity correlated positively with the grade of epithelial dysplasia in the total cohort of pregnant and non-pregnant patients; expression increased linearly from CIN1 to CIN3. Furthermore, the association between p16 immunostaining and CIN grade was significant in non-pregnant patients but not in pregnant patients. In pregnant patients, positivity for Ki-67 was less intense than in non-pregnant patients. These results appear
\end{abstract}

Correspondence to: Professor Andrea Ciavattini, Gynaecologic Section, Woman's Health Sciences Department, Marche Polytechnic University, 11 Via Filippo Corridoni, I-60123 Ancona, Italy

E-mail: ciavattini.a@libero.it

Key words: pregnancy, cervical intraepithelial neoplasia, p16, Ki-67 antigen, human papilloma virus to suggest that pregnancy status interferes with the expression of cellular proteins involved in cell-cycle regulation and the carcinogenic process induced by high-risk human papilloma virus, exhibiting increased variability in their staining.

\section{Introduction}

The results of Pap tests performed during routine screenings at the beginning of prenatal care are abnormal in $8-12 \%$ of cases (1). Overall, the prevalence of abnormal cervical cytology in pregnancy is similar to that of age-matched, non-pregnant women (2). The incidence of cervical intraepithelial neoplasia (CIN) in pregnancy varies among different patient populations, as it does in non-pregnant women, but when age-matched, the risk of CIN is not higher than that among women who are not pregnant, ranging between 3.4 and $10.0 \%$ (3). The management of pregnant women with abnormal cytology depends on the degree of cytological abnormality, the outcome of colposcopy, and, when necessary, directed biopsy. Since, the only diagnosis that may alter management in pregnancy is invasive cancer, the management of pregnant women with abnormal cervical cytology or biopsy-proven CIN is generally more conservative compared with management of similar cytology and histology in non-pregnant women. However, management guidelines for cervical dysplasia are not well defined and are based on data collected from non-pregnant women, the opinion of experts, anecdotal experiences or retrospective series of pregnant women.

Previous studies show a varying postpartum regression rate of CIN of $12-97 \%$ and a persistence/progression rate of 2-60\%; however, there are no definitive data or evidence-based guidelines available for cervical dysplasia diagnosed in pregnancy (4-8). Functional biomarkers, such as Ki-67, p16, p53 and cytokeratin 13/14, have proven useful in the prediction of regressing and non-regressing CIN2-3 lesions. Ki-67 is a non-histone protein that exists as two isoforms encoded by cDNA sequences of 11.5 and $12.5 \mathrm{~kb}$ organized over 15 exons and localized on chromosome 10 (9). Its expression is applied to assess the growth fraction of a cell population (10). p16 is a cellular protein encoded by a gene on chromosome $9 \mathrm{p} 21$. In 

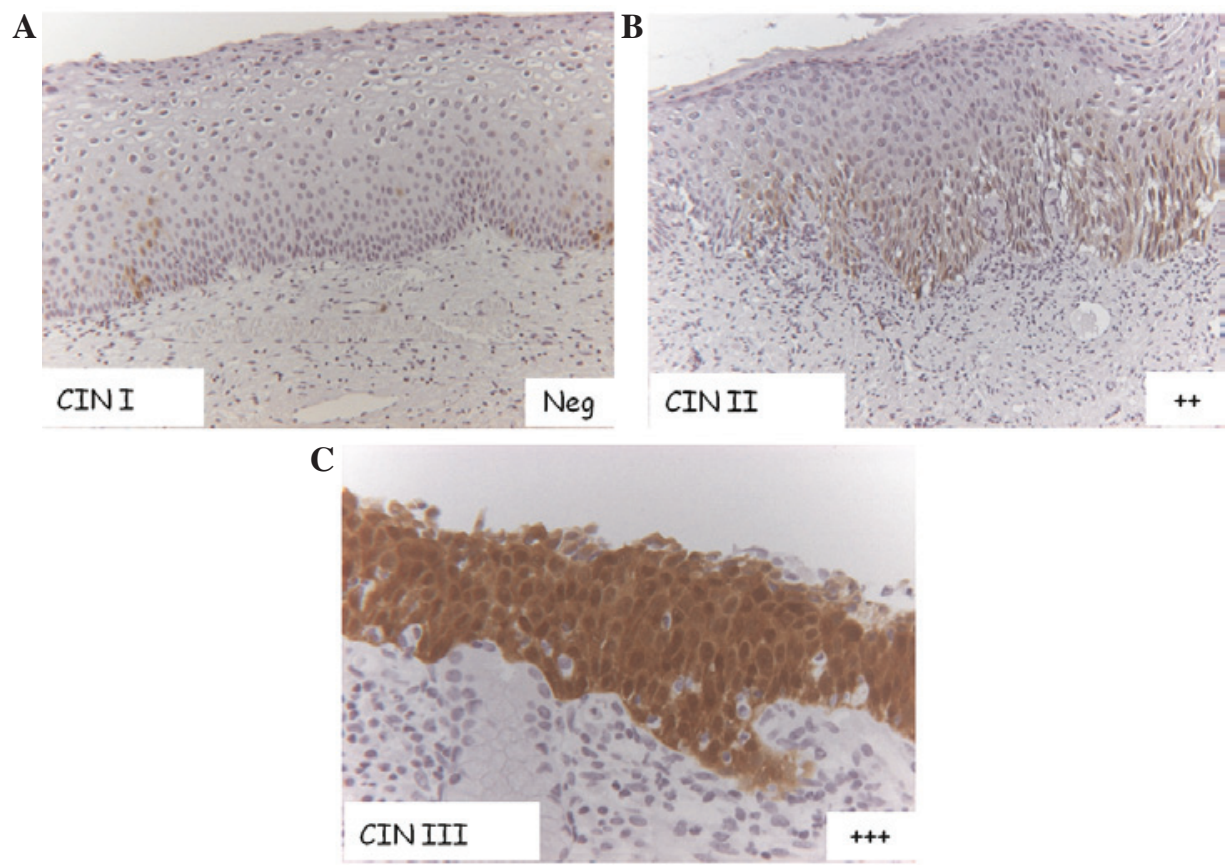

Figure 1. p16 immunostaining in CIN. (A) Complete lack of immunostaining or positivity confined to the lower third of the squamous epithelium (-/+) in CIN1 (magnification, x10). (B) Positivity confined up to two thirds of squamous epithelium (++) in CIN2 (magnification, x10). (C) Positivity is diffused in all epithelial levels (+++) in CIN3 (magnification, x20). CIN, cervical intraepithelial neoplasia.

cervical cancer, p16 expression is correlated with increased expression of oncogenic E6/E7 human papilloma virus (HPV) mRNA (11,12).

Based on the aforementioned data, the present study evaluated Ki-67 and p16 immunostaining in CIN lesions diagnosed in pregnancy compared with those diagnosed in non-pregnant women. The aim was to identify any differences in order to better understand the behavior of CIN in pregnancy.

\section{Patients and methods}

Patients. The present retrospective case-control study included 17 pregnant patients with first-time onset of CIN that were consecutively referred to the affiliated outpatient services of the Lower Genital Tract Disease at the Woman's Health Sciences Department, Gynecologic Section of the Woman's Health Sciences Department, Marche Polytechnic University (Ancona, Italy) and the Gynecological Oncology Unit, Department of Surgical Oncology, Oncologic Referral Centre, National Cancer Institute (Pordenone, Italy) between January 2010 and December 2010. The inclusion criteria were as follows: i) First diagnosis of CIN occurred prior to the 16th gestational week and ii) pregnancies were not obtained by assisted reproductive technologies. Exclusion criteria included: i) Previous cervical treatment (not only for HPV-associated disease); ii) immunocompromised status; iii) chronic hepatitis B and/or C; and iv) cigarette smoking.

The control group included 17 non-pregnant patients with first-time onset of CIN that were consecutively referred to the same institutes during the same study period, and matched for age, parity and number of previous sexual partners. The control group also complied with the same exclusion criteria.
Ethics statement. The present study was designed as a basic scientific research study and, therefore, did not require approval from a Research Ethics Committee. Approval was obtained from the Ethics Committee of Marche Polytechnic University in order to routinely collect data. Written informed consent for the use of personal data was obtained from each patient.

Immunohistochemistry. Following colposcopy, punch cervical biopsies were obtained from the transformation zone and fixed in 10\% buffered formaldehyde (Diapath S.P.A., Martinengo, Italy), embedded in paraffin (AppliChem $\mathrm{GmbH}$, Darmstadt, Germany), sectioned to a thickness of 5-6 $\mu \mathrm{m}$ and stained with hematoxylin-eosin (Bio-Optica S.P.A., Milano, Italy) for routine histological examination.

The sections were dewaxed in xylene (Carlo Erba Reagents, Val-de-Reuil, France) and rehydrated through a graded series of ethanol (Carlo Erba Reagents). The p16 mouse monoclonal antibody (clone E6H4; ready-to-use) was included in the CINtec ${ }^{\circledR}$ Histology kit (catalog no. 9517; Ventana Medical Systems, Inc., Heidelberg, Germany), which was used according to the manufacturer's protocol.

The Ki-67 mouse monoclonal antibody (clone MIB-1; catalog no. M7240; Dako, Golstrup, Denmark) was used at a 1:80 dilution in Antibody Diluent (Dako). Briefly, to better enhance antigenic sites, a low pH Dako Target Retrieval solution (catalog no. K8005) was applied, by incubating sections in a Dako PT-Link autostainer at $750 \mathrm{Kw}$ for $20 \mathrm{~min}$.

Endogenous peroxidase activity was quenched by incubating the sections in 3\% (v/v) hydrogen peroxide (Dako) for $7 \mathrm{~min}$ at room temperature. Tissue sections were incubated with the anti-Ki-67 monoclonal antibody for $60 \mathrm{~min}$. The antigen-antibody complex was subsequently detected using the 
Dako EnVision ${ }^{\mathrm{TM}}$ Detection System, Peroxidase/DAB (catalog no. K801021; Dako). Sections were counterstained with Mayer's haematoxylin (Bio-Optica S.P.A.) and cover-slipped with Eukitt (Bio-Optica S.P.A.).

All histological specimens with stained with hematoxylin-eosin were initially examined by a pathologist to assess the grade of CIN (CIN1/2/3), using a Nikon Eclipse E800 light microscope (Nikon Italia, Firenze, Italy). The evaluation of p16 and Ki-67 positive cells on cervical biopsy sections was performed by light microscopy at appropriate magnifications. Immunoreactivity for the antibody was evaluated by two observers and experiments were repeated three times; discordant results were reconsidered in a consensus review. The cells considered positive exhibited nuclear and/or cytoplasmic staining for p16 and nuclear staining for $\mathrm{Ki}-67$. Immunoreactivity was evaluated using a semi-quantitative three point-scale system, as it follows: i) $-/+$, if there was a complete lack of immunostaining or if positivity was confined to the lower third of the squamous epithelium; ii) ++ , if positivity was confined to less than two thirds of the squamous epithelium; iii) +++ , if reactivity was observed in all epithelial levels, regardless of the staining intensity (Fig.1).

Statistical analysis. Data were analyzed using STATA software (version 11; StataCorp LP, College Station, TX, USA). Fisher's exact test was used to assess the association between categorical variables. Experiments were performed twice. $\mathrm{P}<0.05$ was considered to indicate a statistically significant difference.

\section{Results}

Characteristics of the population. Of the 34 patients involved in the present study, 4 were classified as CIN1 (25\% of cases; $75 \%$ of controls), 13 as CIN2 (70\% of cases; $30 \%$ of controls) and 17 as CIN3 (35\% of cases; $75 \%$ of controls). There were no statistically significant differences in CIN grade distribution between pregnant and not-pregnant patients. The mean age of the women was the same in both groups (mean \pm standard deviation, $34.6 \pm 4.8$ years). Cases and controls were also comparable for parity, smoking status and number of previous sexual partners (data not shown). p16 and Ki-67 expression in the cervical biopsies and their association with CIN grade in the entire study population were evaluated (Tables I and II).

p16 expression. By analyzing p16 immunoreactivity, negative staining or positive staining confined at the lower third of the squamous epithelium (-/+) was observed in 9 patients. Diffuse positive staining was observed in significantly more CIN2/3 samples than CIN1 samples $(\mathrm{P}=0.008)$. In particular, a complete lack of immunostaining or positivity confined to the lower third of the squamous epithelium $(-/+)$ was observed in $100.0 \%(4 / 4)$ of CIN1, 15.4\% (2/13) of CIN2 and $17.6 \%$ (3/17) of CIN3 samples; positivity confined to less than two thirds of the squamous epithelium (++) was observed in $0.0 \%$ (0/4) of CIN1, $46.1 \%(6 / 13)$ of CIN2 and $17.6 \%$ (3/17) of CIN3 samples; and diffuse positivity in all epithelial levels $(+++)$ was observed in $0.0 \%(0 / 4)$ of CIN1, 38.5\% (5/13) of CIN2 and $64.8 \%$ (11/17) of CIN3 samples (Table I). In CIN3, diffuse
Table I. Correlation between p16 expression and CIN grade in the total study population $(\mathrm{P}=0.008)$.

\begin{tabular}{llcr}
\hline & \multicolumn{3}{c}{ Patients, n (\%) } \\
\cline { 2 - 4 } p16 expression & \multicolumn{1}{c}{ CIN1 } & CIN2 & \multicolumn{1}{c}{ CIN3 } \\
\hline$-/+$ & $4(100.0)$ & $2(15.4)$ & $3(17.6)$ \\
++ & $0(0.0)$ & $6(46.1)$ & $3(17.6)$ \\
+++ & $0(0.0)$ & $5(38.5)$ & $11(64.8)$ \\
Total & $4(100.0)$ & $13(100.0)$ & $17(100.0)$ \\
\hline
\end{tabular}

CIN, cervical intraepithelial neoplasia.

Table II. Correlation between Ki-67 expression and CIN grade in the total study population $(\mathrm{P}<0.001)$.

\begin{tabular}{llcc}
\hline & \multicolumn{3}{c}{ Patients, n(\%) } \\
\cline { 2 - 4 } Ki-67 expression & CIN1 & CIN2 & CIN3 \\
\hline$-/+$ & $4(100.0)$ & $3(23.1)$ & $0(0.0)$ \\
++ & $0(0.0)$ & $7(53.8)$ & $8(47.1)$ \\
+++ & $0(0.0)$ & $3(23.1)$ & $9(52.9)$ \\
Total & $4(100.0)$ & $13(100.0)$ & $17(100.0)$ \\
\hline
\end{tabular}

CIN, cervical intraepithelial neoplasia.

positivity for p16 (+++) was observed in $72.7 \%$ of non-pregnant women, but only in $50.0 \%$ of pregnant women. Positivity for Ki-67 was confined at the lower third of the epithelium or complete absent (-/+) in $100 \%(4 / 4)$ of CIN1, $23.1 \%(3 / 13)$ of CIN2 and $0 \%(0 / 17)$ of CIN3; positivity confined to less than two thirds of the epithelium $(++)$ was observed in $0 \%(0 / 0)$ of CIN1, 53.8\% (7/13) of CIN2 and $47.1 \%$ (8/17) of CIN3; and diffuse positive immunostaining $(+++)$ was observed in $0 \%$ $(0 / 4)$ of CIN1, 23.1\% (3/13) of CIN2 and 52.9\% (9/17) of CIN3 (Table II). Statistical analysis revealed that the association between p16 immunostaining and CIN grade was significant in non-pregnant patients $(\mathrm{P}=0.003)$ but not in pregnant patients $(\mathrm{P}=0.344)$ (Table III).

Ki-67 expression. Positivity for Ki-67 was less intense in pregnant patients than in non-pregnant patients. Ki-67 was expressed in all epithelial levels in $16.7 \%$ of pregnant patients with CIN3 compared with $72.7 \%$ of non-pregnant patients with CIN3 (Table IV). Ki-67 immunostaining was significantly associated with CIN grade in non-pregnant patients $(\mathrm{P}=0.003)$ but not in pregnant patients $(\mathrm{P}=0.236)$.

Association between pl6 and Ki-67 immunoreactivity. The expression of the two biomarkers was compared, revealing an association with the CIN grade $(\mathrm{P}=0.002)$ in the total patient cohort. The association between Ki-67 and p16 expression persisted when the analysis was narrowed exclusively to pregnant women $(\mathrm{P}=0.019)$ (Table $\mathrm{V})$, but not in non-pregnant women (data not shown). 
Table III. Correlation between p16 expression and CIN grade in pregnant (cases) ( $\mathrm{P}=0.344)$ and in non-pregnant (controls) $(\mathrm{P}=0.003)$ women.

\begin{tabular}{|c|c|c|c|c|c|c|}
\hline \multirow[b]{2}{*}{ p16 expression } & \multicolumn{2}{|c|}{ CIN1, n (\%) } & \multicolumn{2}{|c|}{ CIN2, n (\%) } & \multicolumn{2}{|c|}{ CIN3, n (\%) } \\
\hline & Cases & Controls & Cases & Controls & Cases & Controls \\
\hline$-/+$ & $1(100.0)$ & $3(100.0)$ & $2(20.0)$ & $0(0.0)$ & $0(0.0)$ & $3(27.3)$ \\
\hline++ & $0(0.0)$ & $0(0.0)$ & $4(40.0)$ & $2(66.7)$ & $3(50.0)$ & $0(0.0)$ \\
\hline+++ & $0(0.0)$ & $0(0.0)$ & $4(40.0)$ & $1(33.3)$ & $3(50.0)$ & $8(72.7)$ \\
\hline Total & $1(100.0)$ & $3(100.0)$ & $10(100.0)$ & $3(100.0)$ & $6(100.0)$ & $11(100.0)$ \\
\hline
\end{tabular}

CIN, cervical intraepithelial neoplasia.

Table IV. Correlation between Ki-67 expression and CIN grade in pregnant (cases) $(\mathrm{P}=0.236)$ and non-pregnant (controls) $(\mathrm{P}=0.003)$ women.

\begin{tabular}{|c|c|c|c|c|c|c|}
\hline \multirow[b]{2}{*}{ Ki-67 expression } & \multicolumn{2}{|c|}{ CIN1,n (\%) } & \multicolumn{2}{|c|}{ CIN2, n (\%) } & \multicolumn{2}{|c|}{ CIN3, n (\%) } \\
\hline & Cases & Controls & Cases & Controls & Cases & Controls \\
\hline$-/+$ & $1(100.0)$ & $3(100.0)$ & $3(30.0)$ & $0(0.0)$ & $0(0.0)$ & $0(0.0)$ \\
\hline++ & $0(0.0)$ & $0(0.0)$ & $5(50.0)$ & $2(66.7)$ & $5(83.3)$ & $3(27.3)$ \\
\hline +++ & $0(0.0)$ & $0(0.0)$ & $2(20.0)$ & $1(33.3)$ & $1(16.7)$ & $8(72.7)$ \\
\hline Total & $1(100.0)$ & $3(100.0)$ & $10(100.0)$ & $3(100.0)$ & $6(100.0)$ & $11(100.0)$ \\
\hline
\end{tabular}

CIN, cervical intraepithelial neoplasia.

\section{Discussion}

Few clinical studies have been performed concerning cervical intraepithelial lesions during pregnancy and data regarding the influence of pregnancy on the natural history of CIN are discordant. However, the risk of progression of CIN $2 / 3$ to invasive cervical cancer during pregnancy appears to be minimal (4-8) however, the rate of spontaneous regression post-partum is relatively high. Spontaneous regression is reported in 12-97\% of cases, while persistence in the severity of CIN is reported in $25-47 \%$ of cases (13-15). Based on this data, it is established that patients with any grade of CIN in pregnancy should be conservatively managed after an invasive disease has been excluded (16). However, this topic continues to have a great clinical relevance, as premalignant lesions are typically diagnosed in the fertile age range and cervical cancer is the most commonly occurring malignancy during pregnancy, with an incidence of 1.2-4.5 per 10,000 women (17).

In the present study, p16 and $\mathrm{Ki}-67$ immunostaining was analyzed in patients diagnosed with CIN lesions during pregnancy compared with those diagnosed in non-pregnant women. The results showed an increased variability in the expression of these biomarkers in pregnant patients.

p16 and Ki-67 were selected for analysis in the present study, as they are two biomarkers with available standardized, commercial assays and have been most commonly evaluated in clinical studies. Overexpression of p16 and Ki-67 have proven to be useful indicators of clinically significant infections and lesion severity in numerous studies $(11,18,19)$; several properties
Table V. Correlation between p16 and Ki-67 expression in pregnant women $(\mathrm{P}=0.019)$.

\begin{tabular}{lccc}
\hline & \multicolumn{3}{c}{ Ki-67 expression, $n$} \\
\cline { 2 - 4 } p16 expression, $n$ & $-/+$ & ++ & +++ \\
\hline-++ & 3 & 0 & 0 \\
++ & 0 & 6 & 1 \\
+++ & 1 & 4 & 2 \\
\hline
\end{tabular}

of p16 and Ki-67 enable them to be promising biomarkers for HPV-associated cancer, since they are associated with histological grade and infection for HR-HPV (17). In addition, p16 immunostaining appears to be a useful adjunctive test in the examination of colposcopically-directed cervical biopsies and in the diagnostic cascade of women investigated for abnormal Papanicolaou smears, due to its capacity to reveal the integration of high-risk HPV DNA into the host cell genome (20).

p16 is a cellular correlate of increased expression of oncogenic E6/E7 HPV mRNA (21-25). Its expression is directly associated with the action of the HPV oncogene, as continuous expression of $\mathrm{E} 7$ is necessary to maintain a malignant phenotype in HPV-associated cancer (26). Several studies have shown that positive p16 immunostaining is significantly associated with CIN2/3 or carcinoma (27-34). Although varying efficacy of p16 immunostaining in CIN2-3 or carcinoma has 
been reported, the majority of studies have reported that p16 immunostaining has a high sensitivity to CIN2/3 or carcinoma (range, 82-100\%), supporting the hypothesis that p16 is a suitable biomarker for CIN2/3 (35). Klaes et al identified p16 expression in $60 \%$ of CIN1 cases, while $40 \%$ had no expression or only focal expression (25). In a study by Benevolo et al, none of the normal cervical tissues analyzed exhibited p16 positive staining, whereas a constant and significant increase in protein overexpression was observed in CIN1 (30\%), CIN2 (90\%), CIN3 (100\%) and carcinoma (100\%) tissues (36). More recently, p16 was evaluated as a prognostic marker of progression and regression in series of prospectively recruited patients with CIN1, suggesting that a negative result for p16 may exclude the possibility of progression during follow-up (37).

The Ki-67 protein is a human nuclear antigen strictly associated with cell proliferation. It is present during all active phases of the cell cycle (G1, S, G2 and mitosis) but is absent in resting cells (G0); therefore, it is used to determine the growth fraction of a given cell population. The fraction of Ki-67-positive tumor cells (the Ki-67 labeling index) is commonly correlated with the clinical course of the disease. Previous studies have shown an association between Ki-67 expression and lesion severity or growth rate, and demonstrated the use of $\mathrm{Ki}-67$ expression in the analysis of vulvar and vaginal lesions caused by HPV $(38,39)$. Therefore, the determination of Ki-67 expression appears to be a relevant complementary examination in the detection and distinction of different lesion grades of the uterine cervix.

Data obtained in the present study revealed that p16 and Ki-67 staining occur in a less deep section of the CIN squamous epithelium in pregnant patients than in non-pregnant patients. In contrast to the consistent positive staining for p16 and Ki-67 in non-pregnant women with CIN2/3, CIN2/3 lesions typically exhibited markedly more variable staining in pregnant women. Similarly, the correlation between p16/Ki-67 expression and the severity of CIN was significant in non-pregnant women but not in pregnant women, where increased p16 and Ki-67 staining according to CIN grade was proportionally lower. These results indicate that the pregnancy status of a patient interferes with the expression of cellular proteins involved in cell-cycle regulation and the carcinogenic process induced by high-risk HPV, with increased variability in staining observed in pregnant women. Although it is not known whether this immunohistochemical variability in p16 and Ki-67 staining is also able to provide information on the evolution of CIN lesions in pregnancy, it is important to identify a suitable interpretation for the aforementioned phenomenon. A possible mechanism is associated with changes in the hormonal status during gestation. p16 and Ki-67 staining may depend on altered transcriptional regulation of the viral E6/E7 oncogenes, which affect almost all the cellular pathways involved in HPV-associated carcinogenesis. Thus, the modulation of p16 and Ki-67 expression may be attributed to increased levels of progesterone, the essential hormone for pregnancy. In fact, progesterone influences the gene expression levels of proteases, transcription factors, cell-adhesion molecules, modulators of vascular activities and regulators of inflammation (40). Furthermore, modulation of the individual immune system performed according to the pregnancy status may have a significant influence on the balance of early oncogenic status.
The present study is important for a number of reasons. To the best of our knowledge, it is the first study to evaluate p16 and Ki-67 expression in pregnant women. Furthermore, it was performed over a short period of time at affiliated medical centers, to ensure the samples were homogeneous, and by two colposcopists highly experienced in evaluating lesions in pregnancy. However, the present study did not consider additional factors possibly associated with the evolution of CIN during pregnancy, such as co-infections other than HPV, including Chlamydia trachomatis, herpes simplex virus and cytomegalovirus. Another limitation of the study is the small sample size.

In conclusion, the findings concerning p16 and Ki-67 expression suggest that pregnant patients may exhibit a less aggressive biological behavior of cervical dysplasia than non-pregnant patients. Further clinical studies should be performed to support these findings and apply them to a 'watchful waiting' strategy for the management of cervical dysplasia during pregnancy. From a scientific point of view, the results of the present study encourage further research to identify other biomarkers to aid in better understanding the clinical evolution of CIN during pregnancy, and the influence of the hormonal gestational pattern and the immune system on the natural history of HPV infection.

\section{References}

1. Wu YM, Wang T, He Y, Song F, Wang Y, Zhu L, Kong WM, Duan W and Zhang WY: Clinical management of cervical intraepithelial neoplasia in pregnant and postpartum women. Arch Gynecol Obstet 289: 1071-1077, 2014.

2. Saslow D, Solomon D, Lawson HW, Killackey M, Kulasingam SL, Cain J, Garcia FA, Moriarty AT, Waxman AG, Wilbur DC, et al; American Cancer Society; American Society for Colposcopy and Cervical Pathology; American Society for Clinical Pathology: American Cancer Society, American Society for Colposcopy and Cervical Pathology, and American Society for Clinical Pathology screening guidelines for the prevention and early detection of cervical cancer. Am J Clin Pathol 137: 516-542, 2012.

3. Fader AN, Alward EK, Niederhauser A, Chirico C, Lesnock JL, Zwiesler DJ, Guido RS, Lofgren DJ, Gold MA and Moore KN: Cervical dysplasia in pregnancy: A multi-institutional evaluation. Am J Obstet Gynecol 203: 113.e1-e6, 2010.

4. Insinga RP, Glass AG and Rush BB: Diagnoses and outcomes in cervical cancer screening: A population-based study. Am J Obstet Gynecol 191: 105-113, 2004.

5. Morimura Y, Fujimori K, Soeda S, Hashimoto T, Takano Y, Yamada H, Yanagida K and Sato A: Cervical cytology during pregnancy-comparison with non-pregnant women and management of pregnant women with abnormal cytology. Fukushima J Med Sci 48: 27-37, 2002

6. Douvier S, Filipuzzi L and Sagot P: Management of cervical intra-epithelial neoplasm during pregnancy. Gynecol Obstet Fertil 31: 851-855, 2003 (In French).

7. Frega A, Scirpa P, Corosu R, Verrico M, Scarciglia ML, Primieri MR, Palazzo A, Iacovelli R and Moscarini M: Clinical management and follow-up of squamous intraepithelial cervical lesions during pregnancy and postpartum. Anticancer Res 27: 2743-2746, 2007.

8. Wright TC Jr, Massad LS, Dunton CJ, Spitzer M, Wilkinson EJ and Solomon D; 2006 ASCCP-Sponsored Consensus Conference: 2006 consensus guidelines for the management of women with abnormal cervical cancer screening tests. J Low Genit Tract Dis 11: 201-222, 2007.

9. Duchrow M, Schlüter C, Key G, Kubbutat MH, Wohlenberg C, Flad HD and Gerdes J: Cell proliferation-associated nuclear antigen defined by antibody Ki-67: A new kind of cell cycle-maintaining proteins. Arch Immunol Ther Exp (Warsz) 43: $117-121,1995$.

10. Scholzen T and Gerdes J: The Ki-67 protein: From the known and the unknown. J Cell Physiol 182: 311-322, 2000. 
11. Tornesello ML, Buonaguro L, Giorgi Rossi P and Buonaguro FM: Viral and cellular biomarkers in the diagnosis of cervical Intraepithelial neoplasia and cancer. Biomed Res Int 2013: 519619, 2013

12. von Knebel Doeberitz M: New molecular tools for efficient screening of cervical cancer. Dis Markers 17: 123-128, 2001.

13. Palle C, Bangsbøll S and Andreasson B: Cervical intraepithelial neoplasia in pregnancy. Acta Obstet Gynecol Scand 79: 306-310, 2000.

14. Vlahos G, Rodolakis A, Diakomanolis E, Stefanidis K, Haidopoulos D, Abela K, Georgountzos V and Michalas S: Conservative management of cervical intraepithelial neoplasia (CIN (2-3)) in pregnant women. Gynecol Obstet Invest 54: 78-81, 2002.

15. Yost NP, Santoso JT, Mcintire DD and Iliya FA: Postpartum regression rates of antepartum cervical intraepithelial neoplasia II and III lesions. Obstet Gynecol 93: 359-362, 1999.

16. Sorosky JI: Cervical carcinoma complicating pregnancy. Postgrad Obstet Gynecol 15: 1-6, 1995.

17. Kaplan KJ, Dainty LA, Dolinsky B, Rose GS, Carlson J, McHale M and Elkas JC: Prognosis and recurrence risk for patients with cervical squamous intraepithelial lesions diagnosed during pregnancy. Cancer 102: 228-232, 2004.

18. Zappacosta R, Colasante A, Viola P, D'Antuono T, Lattanzio G, Capanna S, Gatta DM and Rosini S: Chromogenic in situ hybridization and p16/Ki67 dual staining on formalin-fixed paraffin-embedded cervical specimens: Correlation with HPV-DNA test, E6/E7 mRNA test, and potential clinical apllications. Biomed Res Int 2013: 453606, 2013.

19. Carozzi F, Confortini M, Dalla Palma P, Del Mistro A, Gillio-Tos A, De Marco L, Giorgi-Rossi P, Pontenani G, Rosso S, Sani C, et al: Use of p16-INK4A overexpression to increase the specificity of human papillomavirus testing: A nested substudy of the NTCC randomised controlled trial. Lancet Oncol 9: 937-945, 2008.

20. Dray M, Russell P, Dalrymple C, Wallman N, Angus G, Leong A, Carter J and Cheerala B: p16(INK4a) as a complementary marker of high-grade intraepithelial lesions of the uterine cervix. I: Experience with squamous lesions in 189 consecutive cervical biopsies. Pathology 37: 112-124, 2005.

21. Mantovani F and Banks L: The human papillomavirus E6 protein and its contribution to malignant progression. Oncogene 20 : 7874-7887, 2001

22. Munger K, Basile JR, Duensing S, Eichten A, Gonzalez SL, Grace $M$ and Zacny VL: Biological activities and molecular targets of the human papillomavirus E7 oncoprotein. Oncogene 20: 7888-7898, 2001.

23. Khleif SN, DeGregori J, Yee CL, Otterson GA, Kaye FJ, Nevins JR and Howley PM: Inhibition of cyclin D-CDK4/CDK6 activity is associated with an E2F-mediated induction of cyclin kinase inhibitor activity. Proc Natl Acad Sci USA 93: 4350-4354, 1996.

24. Sano T, Oyama T, Kashiwabara K, Fukuda T and Nakajima T: Expression status of p16 protein is associated with human papillomavirus oncogenic potential in cervical and genital lesions. Am J Pathol 153: 1741-1748, 1998.

25. Klaes R, Friedrich T, Spitkovsky D, Ridder R, Rudy W, Petry U, Dallenbach-Hellweg G, Schmidt D and von Knebel Doeberitz M: Overexpression of p16(INK4A) as a specific marker for dysplastic and neoplastic epithelial cells of the cervix uteri. Int J Cancer 92: 276-284, 2001

26. von Knebel Doeberitz M, Rittmüller C, zur Hausen H and Dürst M: Inhibition of tumorigenicity of cervical cancer cells in nude mice by HPV E6-E7 anti-sense RNA. Int J Cancer 51: 831-834, 1992
27. Kong CS, Balzer BL, Troxell ML, Patterson BK and Longacre TA: p16INK4A immunohistochemistry is superior to HPV in situ hybridization for the detection of high-risk HPV in atypical squamous metaplasia. Am J Surg Pathol 31: 33-43, 2007.

28. Walts AE and Bose S: p16, Ki-67 and BD ProExC immunostaining: A practical approach for diagnosis of cervical intraepithelial neoplasia. Hum Pathol 40: 957-964, 2009.

29. Pinto AP, Schlecht NF, Woo TY, Crum CP and Cibas ES: Biomarker (ProEx C, p16INK4A, and MiB-1) distinction of high-grade squamous intraepithelial lesion from its mimics. Mod Pathol 21: 1067-1074, 2008.

30. Badr RE, Walts AE, Chung F and Bose S: BD ProEx C: A sensitive and specific marker of HPV-associated squamous lesions of the cervix. Am J Surg Pathol 32: 899-906, 2008.

31. Shi J, Liu H, Wilkerson M, Huang Y, Meschter S, Dupree W, Schuerch C and Lin F: Evaluation of p16INK4a, minichromosome maintenance protein 2, DNA topoisomerase IIalpha, ProEX C, and p16INK4a/ProEX C in cervical squamous intraepithelial lesions. Hum Pathol 38: 1335-1344, 2007.

32. Branca M, Ciotti M, Santini D, Di Bonito L, Giorgi C, Benedetto A, Paba P, Favalli C, Costa S, Agarossi A, et al: p16(INK4A) expression is related to grade of CIN and high-risk human papillomavirus but does not predict virus clearance after conization or disease outcome. Int J Gynecol Pathol 23: 354-365, 2004.

33. Carreon JD, Sherman ME, Guillén D, Solomon D, Herrero R, Jerónimo J, Wacholder S, Rodríguez AC, Morales J, Hutchinson M, et al: CIN2 is a much less reproducible and less valid diagnosis than CIN3: Results from a histological review of population-based cervical samples. Int J Gynecol Pathol 26: 441-446, 2007.

34. Baak JP, Kruse AJ, Janssen E and van Diermen B: Predictive testing of early CIN behaviour by molecular biomarkers. Cell Oncol 27: 277-280, 2005.

35. Roelens J, Reuschenbach M, von Knebel Doeberitz M, Wentzensen N, Bergeron C and Arbyn M: p16INK4a immunocytochemistry versus human papillomavirus testing for triage of women with minor cytologic abnormalities: A systematic review and meta-analysis. Cancer Cytopathol 120: 294-307, 2012.

36. Benevolo M, Terrenato I, Mottolese M, Marandino F, Muti P, Carosi M, Rollo F, Ronchetti L, Mariani L, Vocaturo G and Vocaturo A: Comparative evaluation of $\mathrm{nm} 23$ and p16 expression as biomarkers of high-risk human papillomavirus infection and cervical intraepithelial neoplasia $2(+)$ lesions of the uterine cervix. Histopathology 57: 580-586, 2010.

37. Pacchiarotti A, Ferrari F, Bellardini P, Chini F, Collina G, Dalla Palma P, Ghiringhello B, Maccallini V, Musolino F, Negri G, et al: Prognostic value of p16-INK4A protein in women with negative or CIN1 histology result: A follow-up study. Int J Cancer 134: 897-904, 2014.

38. Sarian LO, Derchain SF, Yoshida A, Vassallo J, Pignataro F and De Angelo Andrade LA: Expression of cycloxygenase-2 (COX-2) and $\mathrm{Ki} 67$ as related to disease severity and HPV detection in squamous lesions of the cervix. Gynecol Oncol 102: 537-541, 2006.

39. Calil LN, Edelweiss MI, Meurer L, Igansi CN and Bozzetti MC: p16 INK4a and Ki67 expression in normal, dysplastic and neoplastic uterine cervical epithelium and human papillomavirus (HPV) infection. Pathol Res Pract 210: 482-487, 2014.

40. Kim J, Bagchi IC and Bagchi MK: Control of ovulation in mice by progesterone receptor-regulated gene networks. Mol Hum Reprod 15: 821-828, 2009. 